\title{
Enhanced Atom Probe Imaging using Generalised Field Evaporation Models
}

Charles Fletcher ${ }^{1}$, Michael Moody ${ }^{1}$, Jeroen Scheerder $^{2}$, Claudia Fleischmann ${ }^{3}$, Brian Geiser ${ }^{4}$ and Daniel Haley $^{5}$

${ }^{1}$ University of Oxford, Oxford, England, United Kingdom, ${ }^{2}$ Imec, Kapeldreef 75, 3001 Leuven, Belgium, Leuven, Vlaams-Brabant, Belgium, ${ }^{3}$ Imec, Kapeldreef 75, 3001 Leuven, Belgium,Quantum solid-state physics group, KU Leuven, Celestijnenlaan 200D, 3001 Leuven, Belgium, Belgium, ${ }^{4}$ CAMECA Instruments Inc., United States, ${ }^{5}$ University of Oxford, England, United Kingdom

For over twenty years, the atom probe reconstruction methods of choice have been projection-based models [1]. Here a mathematical projection between a hemispherical specimen apex and detector plane is considered to approximate the trajectories of evaporated ions. In reality, the differing field evaporation behaviour of phases in multiphase material systems can result in the specimen apex deviating away from the assumed hemispherical shape. This in turn causes the break down of the projection models' simplistic assumptions, introducing spatial distortions of ions within projection-based reconstructions. While a number of modifications to these mathematical projection models have been proposed in an attempt to correct certain aspects of these distortions $[2,3]$, the vast majority of multiphase distortions still remain. More novel reconstruction protocols have also been proposed. However, these typically still impose unphysical geometric constraints on the evolving specimen geometry or are practically too slow for reconstruction of experimental data $[4,5,6]$. In order to truly understand and fully correct for these distortions, sufficiently accurate yet computationally efficient models of specimen-specific evaporation are required.

In this presentation we discuss further developments to CASRA 3D [7], a simulation tool for predicting specimen evaporation and the charged optics of evaporated ions. Unlike previous atomic-scale modelling techniques, our model approximates the specimen surface as a continuum through a level set method. Electrostatics are efficiently solved via a Boundary Element Method. While unable to capture the finer scale complexity of atomic scale evaporation effects [8], this continuum approximation enables simulation of full-scale specimen evaporation in minutes on standard computing hardware. Simulation of the true specimen geometries matching those in experiment can also be achieved via model initialisation directly from electron tomography data (see Figure 1a).

By sampling ion trajectories over the specimen surface, we construct a forward trajectory mapping between the specimen surface and detector that is specific to a particular specimen model. Local image magnification and regions of spatial trajectory overlap can be calculated directly from this mapping (see Figure 1b). By also considering the local evaporation rate of ions over the specimen surface, we can rapidly predict density and composition detector maps that are directly comparable to experiment, without having to launch realistic numbers of ions. Finally, we can also estimate a time-dependent inverse trajectory mapping from detector positions onto the evolving specimen surface, enabling model driven reconstruction [7].

In order to produce an accurate image reconstruction, the unknown parameters of our evaporation model must be constrained from the experimental data. This can be achieved by tuning these parameters to ensure maximum correlation between the model phases and the generated reconstruction's composition via a Mutual Information (MI) metric. The applicability of diffeomorphic metric mappings, typically applied 
in computational anatomy to align image data onto a model organ, will also be considered for precise alignment of the specimen model at interfaces.

Through model optimisation, the calibration of model driven reconstructions for multiple experimental semiconductor datasets will be demonstrated. These calibrated reconstructions successfully reduce multiphase spatial distortions compared to traditional projection-based methods (see Figure 1c-e), promising enhanced data analysis capabilities.

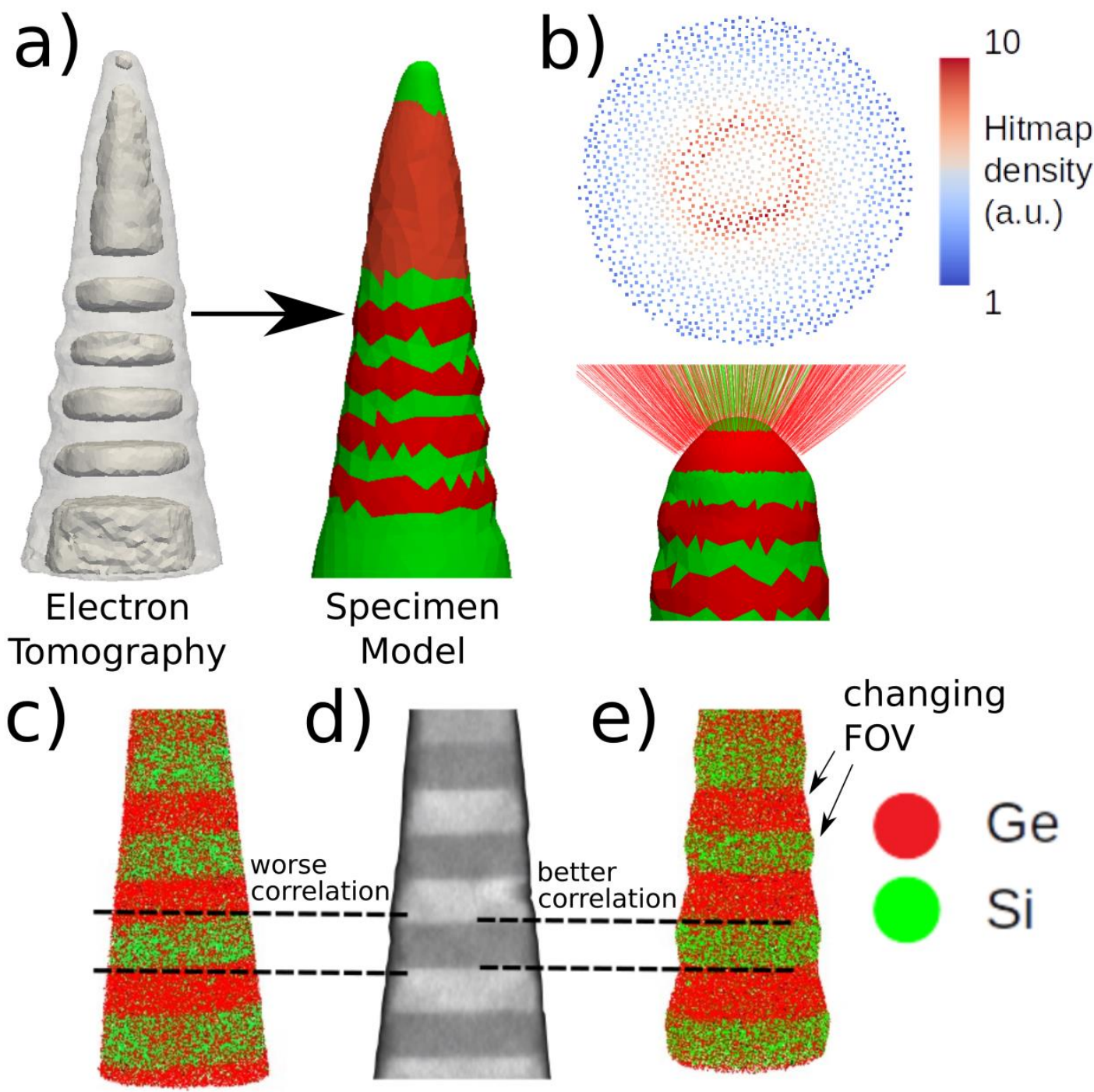

Figure 1. (a) Initialisation of specimen model for a multilayer semiconductor structure from electron tomography (data provided by Imec [9]). (b) Calculation of density detector map midway through the simulated evaporation. (c) Traditional projection-based atom probe reconstruction of the same structure. Note the tilting of the green Si layers. (d) Electron tomography cross-section of the same specimen. (e) Model driven reconstruction of the same dataset. Through model calibration and alignment via mutual information maximisation, layer tilting has been successfully corrected for. 


\section{References}

[1] P. Bas et al, Appl Surf Sci. 87/88 (1995), p. 298-304.

[2] B. Gault et al, Ultramicroscopy. 111 (2011), p. 1619-1624.

[3] D. J. Larson et al, Journal of Microscopy. 243 (2011), p. 15-30.

[4] D. Beinke et al, Ultramicroscopy. 165 (2016), p. 34-41.

[5] N. Rolland et al, Microsc Microanal. 23 (2017), p. 247-254.

[6] D. Beinke et al, Nanoscale. 12 (2020). p. 2820-2832.

[7] C. Fletcher et al, J. Phys. D: Appl. Phys. 53 (2020) 475303.

[8] C. Oberdorfer et al, Materials Characterisation. 146 (2018). p. 324-335.

[9] The authors thank H. Bender, P. Kundu and O. Richard (Imec) for the TEM tomography, and M. Dialameh (Imec) for the APT data shown in Figure 1.

[10] Charles Fletcher acknowledges financial support from CAMECA for this research. 